\title{
Conformational Study and Chiroptical Properties of Chiral Dimethyl- Ethylenedithio-Tetrathiafulvalene (DM-EDT-TTF)
}

\author{
Thomas Cauchy ${ }^{\star a}$, Flavia Pop ${ }^{a}$, Jérôme Cuny ${ }^{b}$, and Narcis Avarvari ${ }^{\star a}$
}

\begin{abstract}
The enantiopure dimethyl-ethylenedithio-tetrathiafulvalene (DM-EDT-TTF) donor exists as biaxial (ax, ax) and biequatorial (eq, eq) conformers in equilibrium. DFT calculations combined with free energy surface (FES) analysis establish that the (ax, ax) form is more stable by $\sim 0.7 \mathrm{kcal} \cdot \mathrm{mol}^{-1}$ than the (eq, eq) one and that the interconversion process involves a sequential conformational change through a boat type (ax, eq) conformer with an activation energy of $\sim 3 \mathrm{kcal}^{\text {. mol }}{ }^{-1}$. TD-DFT calculations show similar, but opposite in sign, CD bands for the two conformers. A Boltzmann type average of the two CD curves, corresponding to a ratio of three (ax, ax) for one (eq, eq) conformers in equilibrium in solution, provides an excellent agreement with the experimental curve.
\end{abstract}

Keywords: Chirality · Circular dichroism · DFT calculations · Free energy surface analysis · Tetrathiafulvalenes
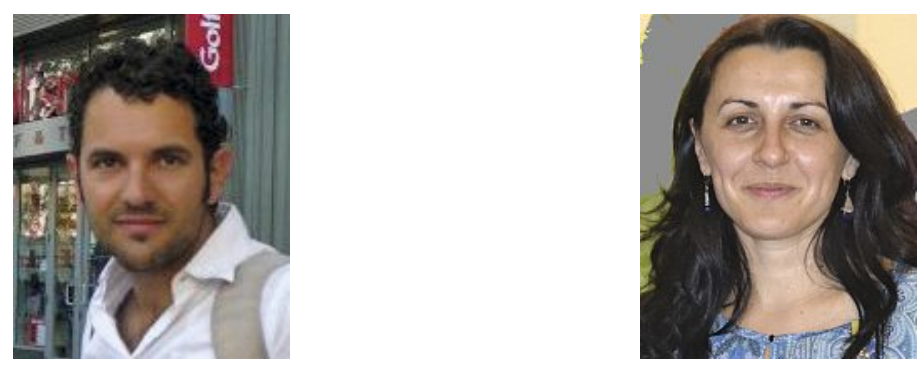

Thomas Cauchy (born in 1980) is a computational chemist at the MOLTECHAnjou laboratory at the University of Angers. He obtained his PhD in 2007 with Eliseo Ruiz at the University of Barcelona. Then he spent two years in a postdoc position in the computational chemistry team of the University of Rennes 1. Finally, after one year as temporary Assistant Professor (ATER) at the University of Angers, he has been appointed Assistant Professor in September 2010 at the same university. His main research interests concern the application of TD-DFT methods for molecular chemistry to model typically reactivity, UV-visible absorption and emission and circular dichroism properties.

${ }^{*}$ Correspondence: Dr. T. Cauchy, Dr. N. Avarvari E-mail: thomas.cauchy@univ-angers.fr, narcis.avarvari@univ-angers.fr aLaboratoire Moltech-Anjou, UMR 6200 CNRSUniversité d'Angers, UFR Sciences, Bât. K 2 Bd. Lavoisier, 49045 Angers, France bLaboratoire de Chimie et Physique Quantiques LCPQ/IRSAMC

Université de Toulouse (UPS) and CNRS

118 Route de Narbonne, F-31062 Toulouse, France
Flavia Pop obtained her $\mathrm{PhD}$ in 2009 (Babeş-Bolyai University, Cluj-Napoca, Romania and University of Angers, France) under the joint supervision of Prof. I. Grosu and Dr. J. Roncali. She has continued her research with a postdoc position (2010-2014) in the field of molecular materials (group of Dr. N. Avarvari), focusing on tetrathiafulvalene-based chiral conducting materials, covalent donor-acceptor systems and chiral supramolecular aggregates, and then a second postdoctoral stay at the University of Nottingham, UK, in the group of Prof. D. Amabilino, working on chiral chromophores and their aggregation for active materials in solar cells. In 2017 she has been appointed CNRS researcher at the Moltech-Anjou Laboratory in Angers.

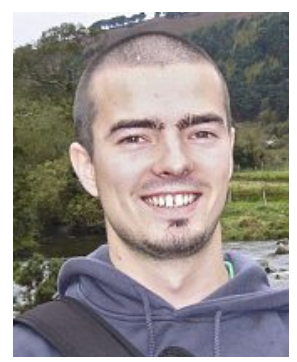

Jérôme Cuny (born in 1984) obtained his $\mathrm{PhD}$ in Chemistry in 2010 under the supervision of Prof. Régis Gautier from the University of Rennes 1. After a twoyear post-doctoral stay in the group of Prof. Michele Parrinello in Lugano (Switzerland), he has been appointed Assistant Professor in chemistry at the University of Toulouse III Paul Sabatier (France) since 2012. His main research interests concern the implementation and application of molecular dynamics approaches to model temperature-dependent structural, dynamical and thermodynamical properties in molecular and solid-state compounds. He is also closely involved in the development of the deMonNano code and the application of the DFTB method to tackle chemical problems. 


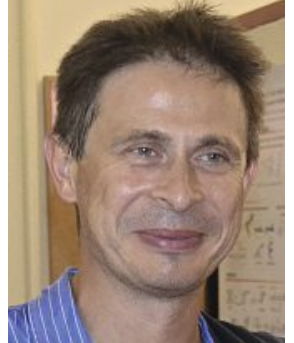

Narcis Avarvari was born in Romania. He received his PhD in Chemistry in 1998 at the Ecole Polytechnique, France. After one-year postdoctoral stay at the ETH Zürich, he obtained in 1999 a permanent research position with the CNRS in Nantes, and then he moved in 2001 to the University of Angers, Laboratory MOLTECH-Anjou. He was awarded with the 2007 Prize of the French Chemical Society, Coordination Chemistry Division and he was promoted CNRS director of research in 2010 . He is currently heading a research team dealing with molecular materials, crystal engineering and coordination chemistry, as well as self-assembly and chirality.

\section{Introduction}

The chiral tetrathiafulvalene (TTF) derivative dimethyl-ethylenedithio-tetrathiafulvalene (DM-EDT-TTF, 1 in Fig. 1) has provided some of the most important results in the field of the chiral conductors, ${ }^{[1]}$ especially through the first experimental observation of the electrical magneto-chiral anisotropy effect (eMChA), ${ }^{[2]}$ a synergetic phenomenon arising in chiral conductors, measured in a magnetic field parallel to the direction of the current, as a consequence of the simultaneous breaking of parity by chirality and of time-reversal symmetry by the magnetic field..[3] The DM-EDT-TTF enantiopure mixed valence radical cation salts showing this effect are formulated as $(R, R)$ - or $(S, S)-(\mathbf{1})_{2} \mathrm{ClO}_{4}$.

Furthermore, by using the series of anions $\mathrm{XF}_{6}^{-}(\mathrm{X}=\mathrm{P}, \mathrm{As}, \mathrm{Sb})$ within the three complete series of salts $(\mathbf{1})_{2} \mathrm{XF}_{6}$, including the racemic and both enantiopure forms, modulation of the packing and, consequently, of the conducting properties with the size of the anion and the nature of the precursor, i.e. racemic versus enantiopure, has been observed. ${ }^{[4]}$ Differences in conductivity between the racemic and enantiopure forms have been noticed as well in radical cation salts derived from TTFoxazoline donors, ${ }^{[5]}$ due to the structural disorder present in the racemic salts, ${ }^{[6]}$ but also in the triiodide salts of the tetramethylated-bis(ethylenedithio)-TTF (TMBEDT-TTF) donor, i.e. the series formulated as (rac)-, $(R, R, R, R)$ - and $(S, S, S, S)$ (TM-BEDT-TTF) $\mathrm{I}_{3} \cdot{ }^{[7]}$ The latter, which is the first reported enantiopure TTF, ${ }^{[8]}$ provided several conducting salts ${ }^{[9]}$ along with its dimethylated counterpart DMBEDT-TTF containing only two stereogenic centers, ${ }^{[10]}$ as in DM-EDT-TTF (1). In these three methylated BEDT and EDT-TTF derivatives an important feature is the conformation of the methyl groups of the dithiin rings ${ }^{[11]}$ as they can adopt either diaxial $(\mathrm{ax}, \mathrm{ax})^{[4 \mathrm{a}, 9 \mathrm{c}, 12,13]}$ or diequatorial (eq, eq $)^{[7]}$ trans arrangements in the chiral precursors (Fig. 1), while in the meso compounds they are necessarily cis (ax, eq). ${ }^{[13]}$ In the corresponding radical cation salts the most often encountered conformation is, by far, the all-equatorial one ${ }^{[2,4,7,9 a-c, 10]}$ but in a very few cases mixed (ax, ax, eq, eq) $)^{[9 c-d, 14]}$ or all-axial[15] conformations have been observed as well for TM-BEDT-TTF derivatives. Clearly, the most important consequence of this conformational issue in the case of the crystalline radical cation salts is for the donor packing, which strongly influences the electron transport properties, while in the case of the neutral precursors different chiroptical properties could possibly arise. Indeed we have previously shown for TMBEDT-TTF that the all-axial conformer is more stable by $1.25 \mathrm{kcal} \cdot \mathrm{mol}^{-1}$ than the all-equatorial one and the experimental circular dicroism (CD) spectra result from the co-existence of both conformers in fast equilibrium in solution, with a predominance of the all-axial one. ${ }^{[7]}$ However the energy surface profile of the equilibrium aimed at identifying transition states and the activation energy of the process was not determined in the previous study. Experimental attempts through solution ${ }^{13} \mathrm{C}$ NMR measurements for the

Fig. 1. $(S, S)$ enantiomer of DM-EDT-TTF (1) with its axial and equatorial conformers.

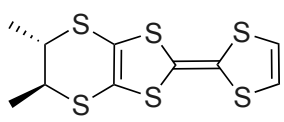

(S,S)-DM-EDT-TTF

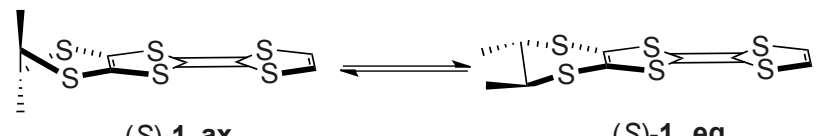

(S)-1 ax

(S)-1 eq estimation of the activation energy of the dimethyl-dihydrodithiin ring allowed only to conclude that this energy barrier was lower than $7.7 \mathrm{kcal} \cdot \mathrm{mol}^{-1} .^{[11]}$ In the present study we have been investigating the conformational equilibrium between the (ax, ax) and (eq, eq) conformers of the DM-EDT-TTF donor 1 through a free energy surface (FES) analysis, determined the lowest energy path for the interconversion of the two energy minima conformers and computed the $\mathrm{CD}$ spectra of the latter.

\section{Results and Discussion}

\subsection{Geometry Optimization and FES Analysis}

Both $(R, R)-\mathbf{1}$ and $(S, S)$-1 enantiomers have been optimized at DFT/PBE1PBE/6$311++\mathrm{G}(3 \mathrm{df}, 2 \mathrm{pd})$ level as (ax, ax) and (eq, eq) conformers (Fig. 2 for $(R, R)-\mathbf{1}$, see the SI for $(S, S)-\mathbf{1})$. In each case the two conformers were energy minima.

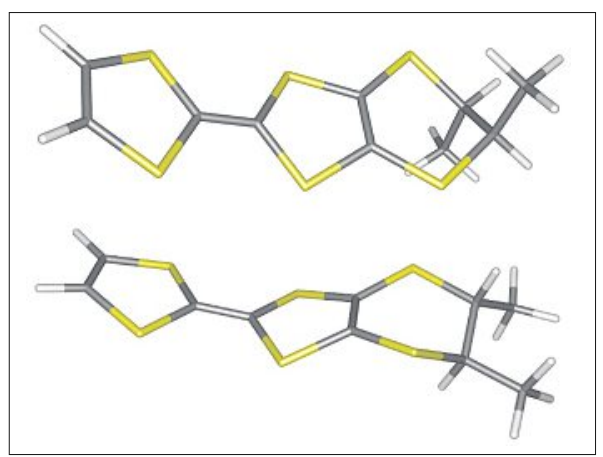

Fig. 2. Optimized geometries for the (ax, ax) (top) and (eq, eq) (bottom) conformers of $(R, R)-\mathbf{1}$.

The calculations clearly indicate a higher stability for the (ax, ax) conformer than the (eq, eq) one for both enantiomers, with an energy difference between them of $\Delta \mathrm{E}=0.675 \mathrm{kcal} \cdot \mathrm{mol}^{-1}$, suggesting a ratio (ax, ax):(eq, eq) of 3.12:1 in the gas phase, which would probably exist in solution as well (vide infra).

To explore the free energy surface (FES) of the $(R, R)-\mathbf{1}$ enantiomer, energies and gradients were calculated using the self-consistent-charge density-functional based tight-binding (SCC-DFTB) approach (see computational details). It is an approximate quantum chemical method derived from DFT and based on a secondorder Taylor expansion of the energy with respect to a reference electronic density. Its parametrization procedure is solely based on DFT calculations. Therefore, it is an excellent choice to compute the whole FES with results that are related to the TD-DFT calculations. Two collective variables were used to explore the FES of the $(R, R)-\mathbf{1}$ enantiomer. They correspond to two dihedral angles and are represented in Fig. 3. 


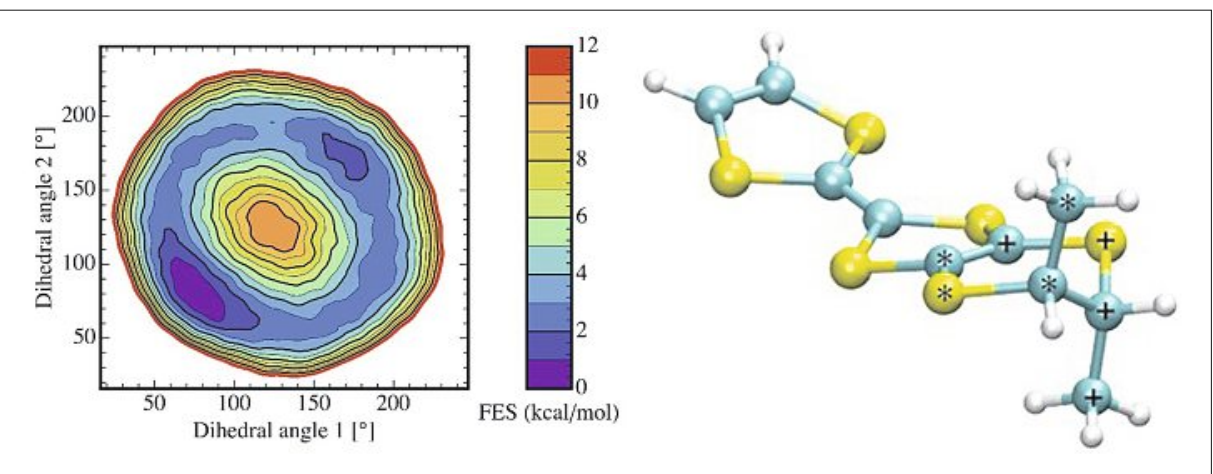

Fig. 3. Free energy surface (FES) of $(R, R)-\mathbf{1}$ obtained from a SCC-DFTB metadynamics simulation performed at $300 \mathrm{~K}$. The two collective variables used to explore the FES are the dihedral angles defined by the stars (angle 1) and by crosses (angle 2). When both angle values are near $180^{\circ}$ it corresponds to the (eq, eq) conformation.

Both dimethyl groups in axial position correspond to the FES minimum and the conversion to the (eq, eq) conformer goes through a mixed conformation with one axial and one equatorial substituent, in which the dihydrodithiin ring adopts very likely a boat conformation. The energy barrier of the process is estimated to be $\sim 3 \mathrm{kcal} . \mathrm{mol}^{-1}$, and DFT geometry optimization of the transition (ax, eq) state gives either the fully axial or fully equatorial conformer. It is worth pointing out that the high freeenergy region located at the center $\left(125^{\circ}, 125^{\circ}\right)$ of the free-energy map prevents a concerted and symmetrical conformational change of both dimethyl groups.

Fig. 4. Experimental and theoretical UVvisible absorption spectra of $(R, R)-\mathbf{1}$. The experimental curve is in blue, whereas the purple and grey curves correspond respectively to the calculated (ax, ax) and (eq, eq) conformers.

\subsection{TD-DFT Calculations}

The theoretical absorption spectra of both conformations are similar and both reproduce the experimental curve (Fig. 4).

Three main transitions are responsible for the UV-vis absorption curve, as highlighted in the following Table for the (ax, ax) conformer (Table 1). All other excitations are detailed in the supplementary information for both conformers.

The first transition has a weak molar absorption coefficient because it is mainly a $\pi$-to- $\sigma$ transition with a weak overlap as (EDD, Fig. 5), where the white hollow surface corresponding to the hole is clearly $\pi$ shown by the electron density difference

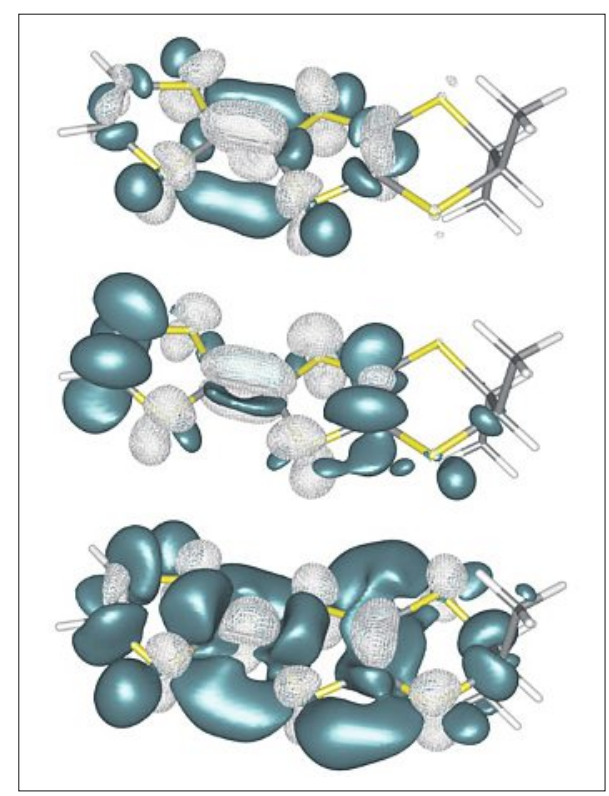

Fig. 5. Electron density difference (EDD) between the excited states $\left(S_{1}, S_{2}\right.$ and $S_{6}$ from top to bottom) and the ground state. The blue and white hollow surfaces represent respectively a gain or a loss of electron density upon excitation. Cut off values correspond to 2.5 $10^{-3}$ electron/bohr ${ }^{3}$.

bonding and sulfur nonbonding, while the blue surfaces representing the excited electron is on the $\sigma$ bond plane. The second process corresponds to a transition from the core $\mathrm{C}=\mathrm{C}$ bonding to both outer $\mathrm{C}=\mathrm{C}$ $\pi^{*}$ bonds. Finally, the $\mathrm{S}_{6}$ excitation is much more complex. It involves all the $\pi$ bonds with quite a strong $\sigma$ participation.

\subsection{Chiroptical Properties}

Considering the chiroptical properties, the relative stability of the (ax, ax) or (eq, eq) conformers is a crucial issue since the two conformations present two radically distinct CD signals (Fig. 6). Clearly, the conformational change does not induce a shift in a band but a change of sign.

If we assume that the experimental conditions (diluted solutions and ambient temperature at atmospheric pressure) can be approximated to the standard conditions and consider $\Delta \mathrm{G}=\Delta_{\mathrm{r}} \mathrm{G}^{\circ}=-\mathrm{RT} \ln \mathrm{K}$, the equilibrium population should be around 3 axial molecules for one equatorial molecule. Indeed a Boltzmann average of the $\mathrm{CD}$ curves provides a good agreement with the experimental curve (Fig. 7).

To simplify the rationale we will focus on the (ax, ax) conformer. In the CD experiment, the first transition is silent (Table 2 ). The first positive band is due to the $S_{2}$ state. The negative band between 30000 $\mathrm{cm}^{-1}$ and $35000 \mathrm{~cm}^{-1}$ is mainly due to the $\mathrm{S}_{4}$ and $\mathrm{S}_{6}$ states and the intense positive change around $35000 \mathrm{~cm}^{-1}$ is due to the $S_{9}$ state. The EDD plots are also indicative of the CD activity, as an important contribution of the asymmetric carbon atoms corre-

\begin{tabular}{|l|l|l|l|}
\hline Excitation & $\mathbf{c m}^{-1}$ & Osc. strength & Monoelectronic excitation description \\
\hline $\mathrm{S}_{0} \rightarrow \mathrm{S}_{1}$ & 21674 & 0.003 & $97 \% \mathrm{HOMO} \rightarrow$ LUMO \\
\hline $\mathrm{S}_{0} \rightarrow \mathrm{S}_{2}$ & 26482 & 0.016 & $93 \% \mathrm{HOMO} \rightarrow$ LUMO+1 \\
\hline $\mathrm{S}_{0} \rightarrow \mathrm{S}_{6}$ & 32474 & 0.246 & complex (involves 4 sets of MO) \\
\hline
\end{tabular}


Fig. 6. Experimental and theoretical $C D$ spectra of $(R, R)-\mathbf{1}$. The experimental curve is in blue, whereas the purple, grey and dotted light blue curves correspond respectively to the calculated (ax, ax), (eq, eq) conformstatistics of 3 (ax, ax) for 1 (eq, eq) conformers. ers and a Boltzmann

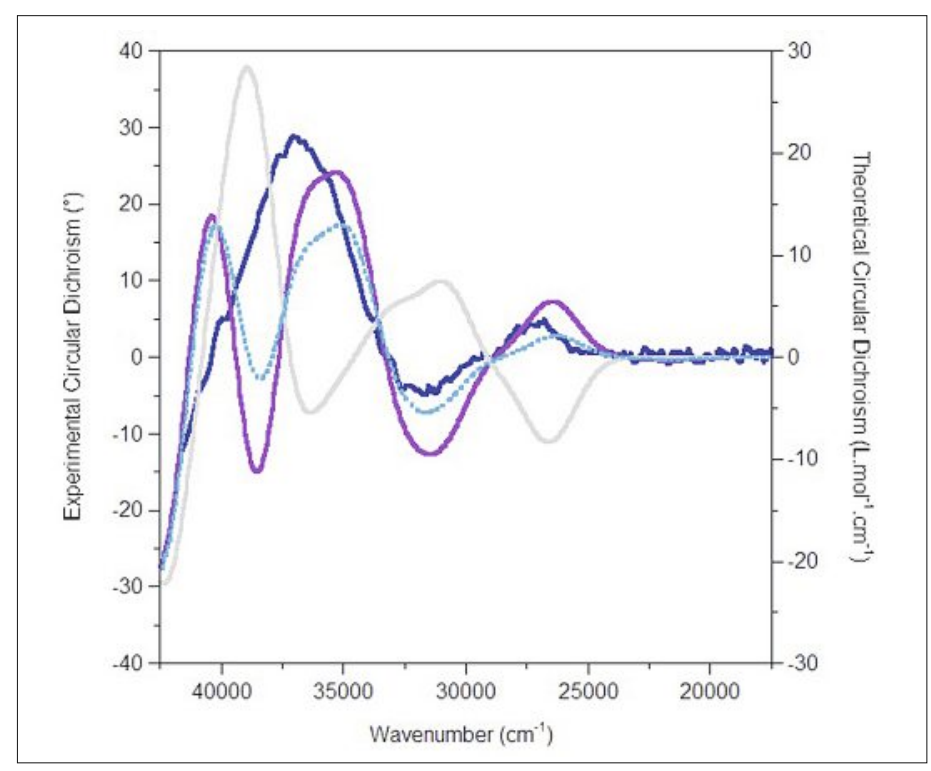

sponds to a more intense CD band. For the transitions where there is no involvement of the electron density of the asymmetric carbons there is no CD signal.

An interesting question is why axial and equatorial signals are opposite in sign, for example, for the first CD band at around $26000 \mathrm{~cm}^{-1}$ although the electron density difference in both cases seems equivalent. The $\mathrm{CD}$ sign depends on the rotation of the electron density upon excitation. In both cases, i.e. (ax, ax) and (eq, eq) conformers, the electron density for the $\mathrm{S}_{0} \rightarrow \mathrm{S}_{2}$ transition comes mainly from the central $\pi$-electrons and goes to the outer $\mathrm{C}=\mathrm{C}$ bonds. The circular dichroism signal arises from the contribution of an asymmetric carbon atom as a small acceptor moiety. The most important difference here, justifying the sign, is the sulfur contribution. This contribution depends on the geometry half chair for the axial molecule or twist boat for the equatorial. As nicely observed in Fig. 8 for the $\mathrm{S}_{0} \rightarrow \mathrm{S}_{2}$ transition, the origin of the hole is centered on the TTF core. Half of the excited electron

Table 2. Most important excited singlet states calculated energies, oscillator and rotatory strengths

\begin{tabular}{|l|l|l|l|}
\hline Excitation & cm $^{-1}$ & $\begin{array}{l}\text { Osc. } \\
\text { strength }\end{array}$ & $\begin{array}{l}\text { Rot. } \\
\text { strength } \\
\text { (in 10-40 } \\
\text { cgs) }\end{array}$ \\
\hline & & & -0.922 \\
\hline $\mathrm{S}_{0} \rightarrow \mathrm{S}_{1}$ & 21674 & 0.003 & 12.694 \\
\hline $\mathrm{S}_{0} \rightarrow \mathrm{S}_{2}$ & 26482 & 0.016 & -16.749 \\
\hline $\mathrm{S}_{0} \rightarrow \mathrm{S}_{4}$ & 30827 & 0.012 & -9.105 \\
\hline $\mathrm{S}_{0} \rightarrow \mathrm{S}_{6}$ & 32474 & 0.246 & -9.087 \\
\hline $\mathrm{S}_{0} \rightarrow \mathrm{S}_{9}$ & 34628 & 0.025 & 28.087 \\
\hline
\end{tabular}

goes to the left outer $\mathrm{C}=\mathrm{C}$ bond whereas the other half goes to the right side, involving the asymmetric carbon atom. Therefore, the slight change of geometry between the conformers has an impact on the overall balance between the left-right repartition. The total electric dipoles of the transition are reported along the EDD in Fig. 8. The change of sign in the CD spectrum can be attributed to the change of orientation of this dipole.

\section{Conclusions}

The relative stability of the two conformers of the chiral dimethyl-ethylenedithio-tetrathiafulvalene (DM-EDT-TTF) donor has been established by gas-phase DFT calculations which indicate that the (ax, ax) conformer is slightly more stable by $\sim 0.7 \mathrm{kcal} \cdot \mathrm{mol}^{-1}$ than the (eq, eq) one. The two conformers are in fast equilibrium as the activation energy of the interconversion process is estimated to $\sim 3 \mathrm{kcal}^{\mathrm{mol}} \mathrm{m}^{-1}$ according to a free energy surface (FES) analysis, the minimum energy path going through an (ax, eq) intermediary. TD-DFT calculations show drastically different chiroptical properties of the two conformers, with the experimental CD curve nicely following a Boltzmann type statistics corresponding to a ratio of 3 (ax, ax) molecules for 1 (eq, eq) one, in agreement with the energy difference between the two conformers. These results bring new insights into the understanding of the chiroptical properties of the dialkyl-ethylene or bis(ethylene)-TTF donors, a very important family of electroactive precursors for chiral conductors.

\section{Experimental and Computational Details}

Circular dichroism spectra were recorded using spectrometric grade solvents in a $0.2 \mathrm{~cm}$ cell at sample concentrations of $10^{-3} \mathrm{M}$ using a JascoJ-815 Circular Dichroism Spectrometer (Biosit facility Université de Rennes 1).

All DFT and TD-DFT calculations have been performed with the Gaussian09 program $^{[16]}$ using the DFT method with the hybrid PBE0 functional (with 25\% of exact exchange) $)^{[17]}$ and the augmented and polarized Pople-type basis set $6-311++\mathrm{G}(3 \mathrm{df}, 2 \mathrm{pd})$. An ultrafine integration grid was chosen. First, the gas-phase ground-state geometries were optimized, starting from the (ax, ax) conformations obtained from the X-ray diffraction data. ${ }^{[4 a]}$ The gas-phase excited-states energies were then determined by a linear response TDDFT method with the same parameters. The length gauge formulation was used for the calculation of the rotatory strengths,

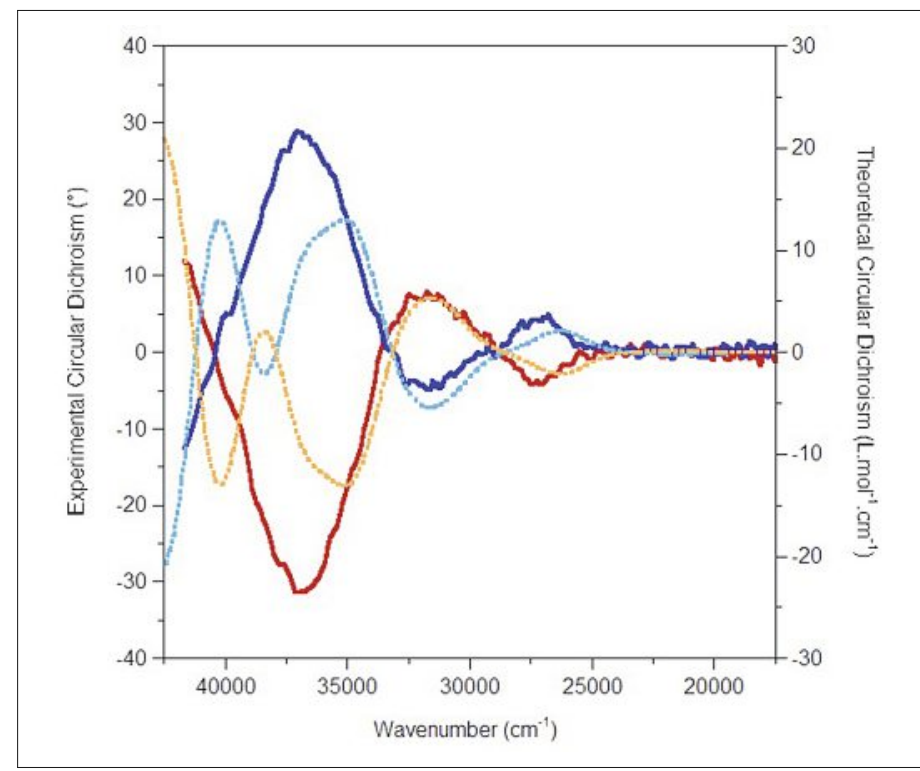

Fig. 7. Experimental and theoretical $C D$ spectra of $(R, R)-\mathbf{1}$ and $(S, S)$-1. Experimental curves are in blue for $(R, R)-1$ and red for $(S, S)-\mathbf{1}$, whereas the dotted light blue and dotted orange curves correspond, respectively, to the calculated Boltzmann statistics of axial and equatorial conformations. 


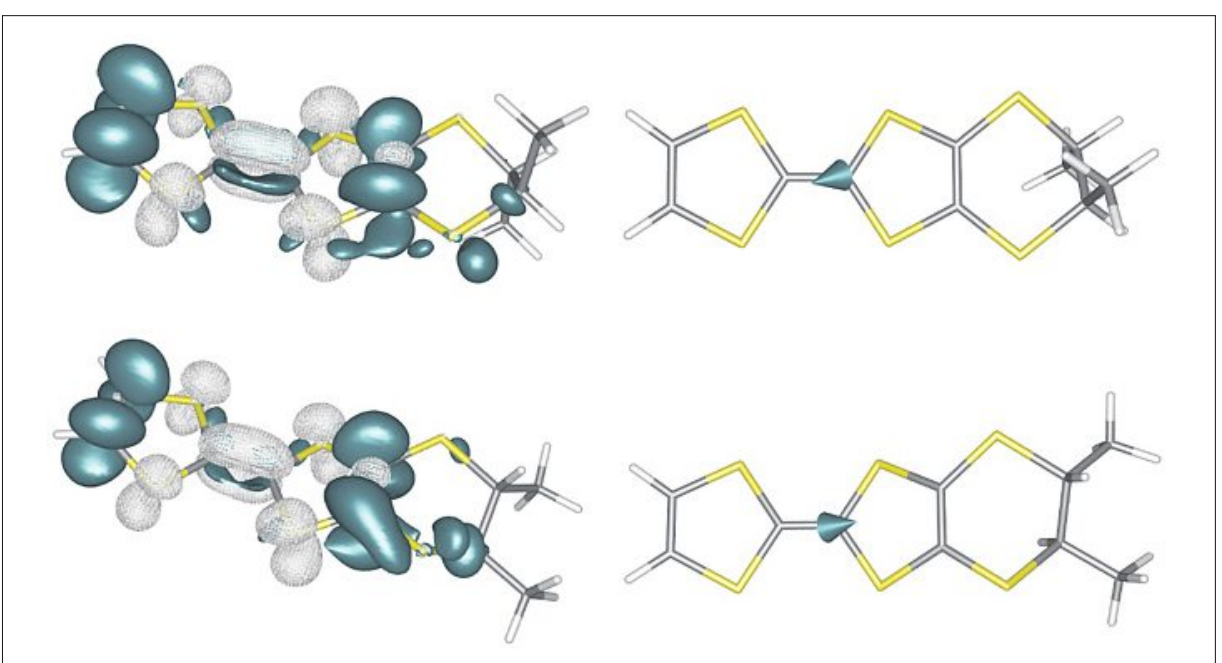

Fig. 8. Electron density difference (EDD) between the excited states $S_{2}$ and the ground state of the (ax, ax) conformation (top left) and the (eq, eq) conformation (bottom left). The blue and white hollow surfaces represent respectively a gain or a loss of electron density upon excitation. The cutoff value was set to $2.510^{-3}$ electron/bohr ${ }^{3}$. The right panels represent the electric dipole moment of the $\mathrm{S}_{0} \rightarrow \mathrm{S}_{2}$ transition for the two conformers.

but both formulas give practically the same values. The calculated spectra have been enlarged by a Gaussian shape [full width at half-maximum $(\mathrm{FWHM})=3000 \mathrm{~cm}^{-1}$ for UV-visible and for CD plots] with GaussSum to compare with the experimental data. ${ }^{[18]}$ The computational results, molecular orbitals and density difference plots were prepared with $\mathrm{ABSiCC}$, a homemade program. ${ }^{[19]}$ The cutoff values were set to represent $50 \%$ of the function.

To explore the free energy surface (FES) of the $(R, R)-\mathbf{1}$ compound, energies and gradients were calculated using the self-consistent-charge density-functional based tight-binding (SCC-DFTB) approach. ${ }^{20]}$ This is an approximate DFT scheme similar to other semiempirical methods whose computational efficiency relies on the use of a minimal valence basisset and parametrized integrals taken from the mio-set of Slater-Koster tables. ${ }^{[20,21]}$ In this formalism, the total energy of the system is written as a sum of three terms: (i) a tight-binding term defined from the aforementioned parametrized integrals; (ii) a repulsive interaction expressed as a sum over all atomic pairs; (iii) the second-order term of the Taylor expansion expressed as a function of the atomic charge fluctuations. The configurational change was investigated using the metadynamics algorithm. ${ }^{[22]}$ Calculations were performed in the canonical ensemble at $300 \mathrm{~K}$. The temperature of the simulation was controlled by a Nosé-Hoover chain of five thermostats with a frequency of $400 \mathrm{~cm}^{-1}$. ${ }^{23]}$ The integration of the equation of motion was achieved with a time step of $0.25 \mathrm{fs}$. In metadynamics, the MD simulation is biased to favor a specific reaction channel adding Gaussian functions to the potential energy surface. In the present study, these
Gaussian functions are defined by height and width of $0.4 \mathrm{kcal}_{\mathrm{mol}} \mathrm{m}^{-1}$ and 0.1 radian, respectively. They were added every 300 MD steps, and we used the well-tempered formulation of metadynamics with a bias factor of 10. ${ }^{[24]}$ All metadynamics simulations were performed with the PLUMED 1.2 package $^{[25]}$ in combination with the deMonNano code.[26]

\section{Acknowledgements}

Financial support from the National Agency for Research (ANR, Project 09-BLAN-0045-01) is gratefully acknowledged. This work was also supported by the CNRS. Biosit facility (Université de Rennes 1) is acknowledged for the access to the CD instrument.

Received: April 15, 2018

[1] N. Avarvari, J. D. Wallis, J. Mater. Chem. 2009, 19,4061

[2] F. Pop, P. Auban-Senzier, E. Canadell, G. L. J. A. Rikken, N. Avarvari, Nat. Commun. 2014, 5, 3757.

[3] a) G. L. J. A. Rikken, J. Fölling, P. Wyder, Phys. Rev. Lett. 2001, 87, 236602-1; b) V. Krstic, S. Roth, M. Burghard, K. Kern, G. L. J. A. Rikken, J. Chem. Phys. 2002, 117, 11315.

[4] a) F. Pop, P. Auban-Senzier, A. Frąckowiak, K. Ptaszyński, I. Olejniczak, J. D. Wallis, E. Canadell, N. Avarvari, J. Am. Chem. Soc. 2013, 135, 17176; b) F. Pop, P. Auban-Senzier, E. Canadell, N. Avarvari, Chem. Commun. 2016, 52,12438 .

[5] a) C. Réthoré, M. Fourmigué, N. Avarvari, Chem. Commun. 2004, 1384; b) C. Réthoré, M. Fourmigué, N. Avarvari, Tetrahedron 2005, 61, 10935 .

[6] a) C. Réthoré, N. Avarvari, E. Canadell, P. AubanSenzier, M. Fourmigué, J. Am. Chem. Soc. 2005, 127, 5748; b) A. M. Madalan, C. Réthoré, M. Fourmigué, E. Canadell, E. B. Lopes, M. Almeida, P. Auban-Senzier, N. Avarvari, Chem. Eur. J. 2010, 16, 528.

[7] F. Pop, S. Laroussi, T. Cauchy, C. J. GómezGarcía, J. D. Wallis, N. Avarvari, Chirality 2013, 25,466 .
[8] J. D. Dunitz, A. Karrer, J. D. Wallis, Helv. Chim. Acta 1986, 69, 69.

[9] a) A. Karrer, J. D. Wallis, J. D. Dunitz, B. Hilti, C. W. Mayer, M. Bürkle, J. Pfeiffer, Helv. Chim. Acta 1987, 70, 942; b) J. R. Galán-Mascarós, E. Coronado, P. A. Goddard, J. Singleton, A. I. Coldea, J. D. Wallis, S. J. Coles, A. Alberola, J. Am. Chem. Soc. 2010, 132, 9271; c) S. Yang, F. Pop, C. Melan, A. C. Brooks, L. Martin, P. Horton, P. Auban-Senzier, G. L. J. A. Rikken, N. Avarvari, J. D. Wallis, CrystEngComm 2014, 16 , 3906; d) M. Atzori, F. Pop, P. Auban-Senzier, R. Clérac, E. Canadell, M. L. Mercuri, N. Avarvari, Inorg. Chem. 2015, 54, 3643.

[10] a) J. S. Zambounis, C. W. Mayer, K. Hauenstein, B. Hilti, W. Hofherr, J. Pfeiffer, M. Bürkle, G. Rihs, Adv. Mater. 1992, 4, 33; b) S. Matsumiya, A. Izuoka, T. Sugawara, T. Taruishi, Y. Kawada, M. Tokumoto, Bull. Chem. Soc. Jpn. 1993, 66, 1949; c) S. J. Krivickas, A. Ichikawa, K. Takahashi, H. Tajima, J. D. Wallis, H. Mori, Synth. Met. 2011, 161, 1563; d) F. Pop, M. Allain, P. Auban-Senzier, J. Martínez-Lillo, F. Lloret, M. Julve, E. Canadell, N. Avarvari, Eur. J. Inorg. Chem. 2014, 3855

[11] S. Matsumiya, A. Izuoka, T. Sugawara, T Taruishi, Y. Kawada, Bull. Chem. Soc. Jpn. 1993 , $66,513$.

[12] F. Pop, N. Avarvari, Beilst. J. Org. Chem. 2015, 11,1105 .

[13] S. Kimura, T. Maejima, H. Suzuki, R. Chiba, H. Mori, T. Kawamoto, T. Mori, H. Moriyama, Y. Nishio, K. Kajita, Chem. Commun. 2004, 2454.

[14] F. Pop, J. Lacour, N. Avarvari, Rev. Roum. Ch. 2012, $57,457$.

[15] F. Pop, P. Batail, N. Avarvari, Crystals 2016, 6, 8

[16] Gaussian 09, Revision A02, Gaussian, Inc., Wallingford CT, 2010, for a full list of authors see Supplementary Information.

[17] a) J. P. Perdew, K. Burke, M. Ernzerhof, Phys. Rev. Lett. 1996, 77, 3865; b) C. Adamo, V. Barone, J. Chem. Phys. 1999, 110, 6158.

[18] N. M. O'boyle, A. L. Tenderholt, K. M. Langner, J. Comput. Chem. 2008, 29, 839.

[19] ABSiCC, Automating Boring Stuff in Computational Chemistry, designed by $\mathrm{T}$. Cauchy and maintained by Y. Morille, University of Angers.

[20] a) M. Elstner, D. Porezag, G. Jungnickel, J. Elsner, M. Haugk, T. Frauenheim, S. Suhai, G. Seifert, Phys. Rev. B 1998, 58, 7260; b) T. Frauenheim, G. Seifert, M. Elsterner, Z. Hajnal, G. Jungnickel, D. Porezag, S. Suhai, R. Scholz, Phys. Status Solidi B 2000, 217, 41; c) P. Koskinen, V. Mäkinen, Comput. Mater. Sci. 2009, 47, 237

[21] T. A. Niehaus, M. Elstner, T. Frauenheim, S Suhai, J. Mol. Struc. (THEOCHEM) 2001, 541 , 185.

[22] a) A. Laio, M. Parrinello, Proc. Natl. Acad. Sci. USA 2002, 99, 12562; b) M. Iannuzzi, A. Laio, M. Parrinello, Phys. Rev. Lett. 2003, 90, 238302; c) A. Laio, A. Rodriguez-Fortea, F. L. Gervasio, M. Ceccarelli, M. Parrinello, J. Phys. Chem. B 2005, 109, 6714

[23] a) S. A. Nosé, J. Chem. Phys. 1984, 81, 511; b) W. G. Hoover, Phys. Rev. A 1985, 31, 1695; c) G. Martyna, M. L. Klein, M. Tuckerman, J. Chem. Phys. 1992, 97, 2635.

[24] A. Barducci, G. Bussi, M. Parrinello, Phys. Rev. Lett. 2008, 100, 020603.

[25] M. Bonomi, D. Branduardi, G. Bussi, C Camilloni, D. Provasi, P. Raiteri, D. Donadio, F. Marinelli, F. Pietrucci, R. A. Broglia, Comput. Phys. Commun. 2009, 180, 1961.

[26] T. Heine, M. Rapacioli, S. Patchkovskii, J. Frenzel, A. Koster, P. Calaminici, H. A. Duarte, S. Escalante, R. Flores-Moreno, A. Goursot, deMonNano; http://demon-nano.ups-tlse.fr/, 2009. 